\title{
Random Attractor of the Stochastic Strongly Damped for the Higher-Order Nonlinear Kirchhoff-Type Equation
}

\author{
Guoguang Lin, Ling Chen, Wei Wang* \\ Department of Mathematics, Yunnan University, Kunming, China \\ Email: gglin@ynu.edu.cn, chen1800828@163.com, *wangw2641@163.com
}

How to cite this paper: Lin, G.G., Chen, L. and Wang, W. (2017) Random Attractor of the Stochastic Strongly Damped for the Higher-Order Nonlinear Kirchhoff-Type Equation. International Journal of Modern Nonlinear Theory and Application, 6, 59-69. http://dx.doi.org/10.4236/ijmnta.2017.62005

Received: April 13, 2017

Accepted: June 11, 2017

Published: June 14, 2017

Copyright ( 92017 by authors and Scientific Research Publishing Inc. This work is licensed under the Creative Commons Attribution International License (CC BY 4.0). http://creativecommons.org/licenses/by/4.0/

\begin{abstract}
In this paper, we consider the stochastic higher-order Kirchhoff-type equation with nonlinear strongly dissipation and white noise. We first deal with random term by using Ornstein-Uhlenbeck process and establish the wellness of the solution, then the existence of global random attractor are proved.
\end{abstract}

\section{Keywords}

Random Dynamical System, Random Attractor, Strongly Dissipation, White Noise

2010 Mathematics Classification: 35K10, 35K25, 35K35

\section{Introduction}

In this paper, we consider the following stochastic strongly damped higherorder nonlinear Kirchhoff-type equation with white noise:

$$
\begin{aligned}
& \mathrm{d} u_{t}+\left[(-\Delta)^{m} u_{t}+\phi\left(\left\|\nabla^{m} u\right\|^{2}\right)(-\Delta)^{m} u+g(u)\right] \mathrm{d} t \\
& =f(x) \mathrm{d} t+q(x) \mathrm{d} W(t), x \in \Omega, m>1,
\end{aligned}
$$

with the Dirichlet boundary condition

$$
u(x, t)=0, \frac{\partial^{i} u}{\partial v^{i}}=0, i=1,2, \cdots, m-1, x \in \partial \Omega,
$$

and the initial value conditions

$$
u(x, 0)=u_{0}(x) \in H^{m}(\Omega), u_{t}(x, 0)=u_{1}(x) \in L^{2}(\Omega),
$$

where $\Omega$ is a bounded domain of $R^{n}$, with a smooth boundary $\partial \Omega, \Delta$ is the Laplacian with respect to the variable $x \in \Omega, u=u(x, t)$ is a real function of $x \in \Omega$ and $t \geq 0, \phi$ is the damping coefficient, $f$ is a given external force, $v$ 
is the outer norm vector, $g(u)$ is a nonlinear forcing, their respectively satisfies the following conditions:

1) $g(u) \leq c_{0}\left(1+|u|^{p}\right), 0<p \leq \frac{2 n}{n-2 m}, n \geq 3$;

2) $\liminf _{t \rightarrow \infty} \frac{s g(s)-c_{1} G(s)}{s^{2}} \geq 0$;

3) $G(s) \geq c_{2}|u|^{p+1}-K_{0}, K_{0}>0$;

4) $\phi(s) \geq m_{0}$;

where $c_{0}, c_{1}, c_{2}, m_{0}$ are positive constants.

As well as we known, the study of stochastic dynamical is more and more widely the attention of scholars, and the study of random attractor has become an important goal. In a sense, the random attractor is popularized for classic determine dynamical system of the global attractor. Global attractor of Kirchhofftype equations have been investigated by many authors, see, e.g., [1] [2] [3] [4], however, the existence random attractor has also been studied by many authors, in [5], Zhaojuan Wang, Shengfan Zhou and Anhui Gu, they study the asymptotic dynamics for a stochastic damped wave equation with multiplicative noise defined on unbounded domains, and investigate the existence of a random attractor, they overcome the difficulty of lacking the compactness of Sobolev embedding in unbounded domains by the energy equation. In [6], Guigui Xu, Libo Wang and Guoguang Lin study the long time behavior of solution to the stochastic strongly damped wave equation with white noise, in this paper, they use the method introduced in [7], so that they needn't divide the equation into two parts. In [8], Zhaojuan Wang, Shengfan Zhou and Anhui Gu study the asymptotic dynamics of the stochastic strongly damped wave equation with homogeneous Neuman boundary condition, and prove the existence of a random attractor. The other long time behavior of solution of evolution equations, we can see [9]-[19].

In this work, we deal with random term by using Ornstein-Uhlenbeck process, the key is to handle the nonlinear terms and strongly damped $(-\Delta)^{m} u_{t}$, and $\phi\left(\left\|\nabla^{m} u\right\|^{2}\right)$ is also difficult to be conducted. So far as we know, there were no result on random attractor for the stochastic higher-order Kirchhoff-type equation with nonlinear strongly dissipation and white noise. It is therefore important to investigate the existence of random attractor on (1.1)-(1.3).

This paper is organized as follows: In Section 2, we recall many basic concepts related to a random attractor for genneral random dynamical system. In Section 3 , we introduce $\mathrm{O}-\mathrm{U}$ process and deal with random term. In Section 4, we prove the existence of random attractor of the random dynamical system.

\section{Preliminaries}

In this section, we collect some basic knowledge about general random dynamical system ([9] [10] [11]).

Let $\left(X,\|.\|_{X}\right)$ be a separable Hilbert space with Borel $\sigma$-algebra $B(X)$. Let $\left(\Omega, F, P,\left(\theta_{t}\right)_{t \in R}\right)$ be the metric dynamical system on the probability space 
$(\Omega, F, P)$.

Definition 2.1. (see [9] [10]). A continuous random dynamical system on $X$ over $\left(\Omega, F, P,\left(\theta_{t}\right)_{t \in R}\right)$ is a $\left(B\left(R^{+}\right) \times F \times B(X), B(X)\right)$-measurable mapping $\varphi: R^{+} \times \Omega \times X \rightarrow X,(t, \omega, u) \mapsto \varphi(t, \omega, u)$. Such that the following properties hold (1)

1) $\varphi(0, \omega,$.$) is the identity on X$;

2) $\varphi(t+s, \omega,)=.\varphi\left(t, \theta_{s} \omega, \varphi(s, \omega,).\right)$ for all $s, t \geq 0$;

3) $\varphi(t, \omega,):. X \rightarrow X$ is continuous for all $t \geq 0$.

Definition 2.2. (see [10])

1) A set-valued mapping $D(\omega): \Omega \rightarrow 2^{X}, \omega \rightarrow D(\omega)$, is said to be a random set if the mapping $\omega \rightarrow d(u, D(\omega))$ is measurable for any $u \in X$. If $D(\omega)$ is also closed (compact) for each $\omega \in \Omega, D(\omega)$ is called a random closed (compact) set. A random set $D(\omega)$ is said to be bounded if there exist $u_{0} \in X$ and a random variable $R(\omega)>0$ such that

$D(\omega) \subset u \in X:\left\|u-u_{0}\right\|_{X} \leq R(\omega)$ for all $\omega \in \Omega$.

2) A random set $D(\omega)$ is called tempered provided for $P$-a.e. $\omega \in \Omega$,

$\lim _{t \rightarrow \infty} \mathrm{e}^{-\beta t} d\left(D\left(\theta_{-t} \omega\right)\right)=0$ for all $\beta>0$, where $d(D)=\sup \|b\|_{X}: b \in D$.

Let $Y$ be the set of all random tempered sets in $X$.

3) A random set $B(\omega)$ is said to be a random absorbing set if for any tempered random set $D(\omega)$, and $P$-a.e. $\omega \in \Omega$, there exists $t_{0}(\omega)$ such that $\varphi\left(t, \theta_{-t} \omega, D\left(\theta_{-t} \omega\right)\right) \subset B(\omega)$ for all $t \geq t_{0}(\omega)$.

4) A random set $B_{1}(\omega)$ is said to be a random attracting set if for any tempered random set $D(\omega)$, and $P$-a.e. $\omega \in \Omega$, we have

$$
\lim _{t \rightarrow \infty} d_{H}\left(\varphi\left(t, \theta_{-t} \omega, D\left(\theta_{-t} \omega\right)\right), B_{1}(\omega)\right)=0,
$$

where $d_{H}$ is the Hausdorff semi-distance given by $d_{H}(E, F)=\sup _{u \in E} \inf _{v \in F}\|u-v\|_{X}$ for any $E, F \subset X$.

5) $\varphi$ is said to be asymptotically compact in $X$ if for $P$ - a.e. $\omega \in \Omega, \varphi\left(t_{n}, \theta_{-t_{n}} \omega, x_{n}\right)_{n=1}^{\infty}$ has a convergent subsequence in $X$ whenever $t_{n} \rightarrow+\infty$, and $x_{n} \in B\left(\theta_{-t_{n}} \omega\right)$ with $B(\omega) \in Y$.

6) A random compact set $A(\omega)$ is said to be a random attractor if it is a random attracting set and $\varphi(t, \omega, A(\omega))=A\left(\theta_{t} \omega\right)$ for $P$-a.e. $\omega \in \Omega$ and all $t \geq 0$.

Theorem 2.1. ([10]) Let $\varphi$ be a continuous random dynamical system with state space $X$ over $\left(\Omega, F, P,\left(\theta_{t}\right)_{t \in R}\right)$. If there is a closed random absorbing set $B(\omega)$ of $\varphi$ and $\varphi$ is asymptotically compact in $X$, then $A(\omega)$ is a random attractor of $\varphi$, where

$$
A(\omega)=\bigcap_{t>0} \overline{\bigcup_{\tau \geq t} \varphi\left(\tau, \theta_{-\tau} \omega, B\left(\theta_{-\tau} \omega\right)\right)}, \omega \in \Omega .
$$

Moreover, $A(\omega)$ is the unique random attractor of $\varphi$.

\section{0-U Process and Stochastic Dynamical System}

Let 


$$
\begin{gathered}
(u, v)=\int_{\Omega} u v \mathrm{~d} x,\|u\|_{0}=(u, u)^{\frac{1}{2}}, \forall u, v \in L^{2}(\Omega), \\
(u, v)_{2 r}=\left(A^{r} u, A^{r} v\right),\|u\|_{2 r}=\left(A^{r} u, A^{r} u\right)^{\frac{1}{2}}, \forall u, v \in V_{2 r}=D\left(A^{r}\right), A=-\Delta .
\end{gathered}
$$

Let $E=H_{0}^{m}(\Omega) \times L^{2}(\Omega)$, and define a weighted inner product and norm in $E$

$$
\begin{gathered}
\left(y_{1}, y_{2}\right)_{E}=\left(\nabla^{m} u_{1}, \nabla^{m} u_{2}\right)+\left(v_{1}, v_{2}\right),\|y\|^{2} E=\left\|\nabla^{m} u\right\|^{2}+\|v\|^{2}, \\
\forall y_{i}=\left(u_{i}, v_{i}\right)^{\mathrm{T}}, \quad y=(u, v)^{\mathrm{T}} \in E, i=1,2 .
\end{gathered}
$$

\subsection{0-U Process}

$\mathrm{O}-\mathrm{U}$ process is given by Wiener process on the metric system $\left(\Omega, F, P,\left(\theta_{t}\right)_{t \in R}\right)$, we can see ([11] [12] [13]).

Let $z\left(\theta_{t} \omega\right)=-\alpha \int_{-\infty}^{0} \mathrm{e}^{\alpha \tau} \theta_{t} \omega(\tau) \mathrm{d} \tau$, where $t \in R$, for $\forall t \geq 0, \quad z\left(\theta_{t} \omega\right)$ meet Itô equation: $\mathrm{d} z+\alpha z \mathrm{~d} t=\mathrm{d} W(t)$. And there is a probability measure $P, \theta_{t}$-invariant set $\Omega_{0} \subset \Omega$; so that stochastic process $z\left(\theta_{t} \omega\right)=-\alpha \int_{-\infty}^{0} \mathrm{e}^{\alpha \tau} \theta_{t} \omega(\tau) \mathrm{d} \tau$ meet the following properties:

1) For $\forall \omega \in \Omega_{0}$, mapping $s \rightarrow z\left(\theta_{s} \omega\right)$ for continuous mapping;

2) Random variable $\|z(\omega)\|$ is called tempered;

3) Exist temper set $r(\omega)>0$, such that

$$
\left\|z\left(\theta_{t} \omega\right)\right\|+\left\|z\left(\theta_{t} \omega\right)\right\|^{2} \leq r\left(\theta_{t} \omega\right) \leq r(\omega) \mathrm{e}^{\frac{\alpha}{2}|t|} ;
$$

4) $\lim _{t \rightarrow+\infty} \frac{1}{t} \int_{0}^{t}\left|z\left(\theta_{t} \omega\right)\right|^{2} \mathrm{~d} \tau=\frac{1}{2 \alpha}$;

5) $\lim _{t \rightarrow+\infty} \frac{1}{t} \int_{0}^{t}\left|z\left(\theta_{t} \omega\right)\right| \mathrm{d} \tau=\frac{1}{\sqrt{\pi \alpha}}$.

\subsection{Stochastic Dynamical System}

For convenience, we rewrite the Question (1.1)-(1.3):

$$
\left\{\begin{array}{c}
\mathrm{d} u=u_{t} \mathrm{~d} t, \\
\mathrm{~d} u_{t}+\left(A^{m} u_{t}+\phi\left(\left\|A^{\frac{m}{2}} u\right\|^{2}\right) A^{m} u+g(u)\right) \mathrm{d} t \\
=f(x) \mathrm{d} t+q(x) \mathrm{d} W(t), t \in[0,+\infty), \\
u(x, 0)=u_{0}(x), u_{t}(x, 0)=u_{1}(x), x \in \Omega .
\end{array}\right.
$$

Let $\psi=(u, y)^{\mathrm{T}}, y=u_{t}+\mu u$, and $\mu=\varepsilon$ ( $\varepsilon$ defined in [20]), then (3.2.1) has the following simple matrix form

$$
\left\{\begin{array}{l}
\mathrm{d} \psi+L \psi \mathrm{d} t=F\left(\theta_{t} \omega, \psi\right), \\
\psi_{0}(\omega)=\left(u_{0}, u_{1}+\mu u_{0}\right)^{\mathrm{T}}
\end{array}\right.
$$

where

$$
\psi=\left(\begin{array}{l}
u \\
y
\end{array}\right), L=\left(\begin{array}{cc}
\mu I & -I \\
\left((\phi-\mu) A^{m}+\mu^{2}\right) I & \left(A^{m}-\mu\right) I
\end{array}\right),
$$




$$
F\left(\theta_{t} \omega, \psi\right)=\left(\begin{array}{c}
0 \\
(-g(u)+f(x)) \mathrm{d} t+q(x) \mathrm{d} W(t)
\end{array}\right) .
$$

Let $v=y-q z\left(\theta_{t} \omega\right)$, then (3.2.1) can be rewritten as the equivalent system:

$$
\left\{\begin{array}{l}
\gamma_{t}+L \gamma=\bar{F}\left(\theta_{t} \omega, \gamma\right), \\
\gamma_{0}(\omega)=\left(u_{0}, u_{1}+\mu u_{0}-q z\left(\theta_{t} \omega\right)\right)^{\mathrm{T}} .
\end{array}\right.
$$

where

$$
\begin{gathered}
\gamma=\left(\begin{array}{l}
u \\
v
\end{array}\right), L=\left(\begin{array}{cc}
\mu I & -I \\
\left((\phi-\mu) A^{m}+\mu^{2}\right) I & \left(A^{m}-\mu\right) I
\end{array}\right), \\
\bar{F}\left(\theta_{t} \omega, \gamma\right)=\left(\begin{array}{c}
q z\left(\theta_{t} \omega\right) \\
-g(u)+f(x)-\left(A^{m}-\mu-1\right) q z\left(\theta_{t} \omega\right)
\end{array}\right) .
\end{gathered}
$$

In [14] [15] they have proven that the operator $L$ of (3.2.3) is the infinitesimal generation operator of $C_{0}$ semigroup $\mathrm{e}^{L t}$ in Hilbert space $E$, $\bar{F}\left(\theta_{t} \omega, \gamma\right):[0,+\infty) \times E \rightarrow E$ is continuous in $t$ and globally Lipschitz continuous in $\gamma$ for each $\omega \in \Omega$. By the classical theory concerning the existence and uniqueness of the solutions [14] [16] [17], so we have the following theorem.

Theorem 3.2.1. Consider (3.2.3). For each $\omega \in \Omega$ and initial value $\gamma_{0}=\left(u_{0}, u_{1}+\mu u_{0}-q z\left(\theta_{t} \omega\right)\right)^{\mathrm{T}} \in E$, there exists a unique function $\gamma$ such that satisfies the integral equation

$$
\gamma(t, \omega)=\mathrm{e}^{-L t} \gamma_{0}+\int_{0}^{t} \mathrm{e}^{L s} \bar{F}(\omega, \gamma(s)) \mathrm{d} s,
$$

and

$$
\gamma \in C\left([0, T) ; H_{0}^{m}(\Omega)\right) \times C\left([0, T) ; L^{2}(\Omega)\right), \forall T>0 .
$$

For $\forall t \geq 0$, a.e. $\omega \in \Omega$, let the solution mapping of $E \rightarrow E$

$$
\tilde{S}(t, \omega): \gamma(0, \omega)=\left(u_{0}(x), v_{0}(x)\right)^{\mathrm{T}} \mapsto \gamma(t, \omega)=(u(x, t, \omega), v(x, t, \omega))^{\mathrm{T}}
$$

generates a random dynamical system.

Define two isomorphic mapping:

$$
\begin{aligned}
T_{\mu}\left(\theta_{t} \omega\right):\left(y_{1}, y_{2}\right)^{\mathrm{T}} & \mapsto\left(y_{1}, y_{2}-\mu y_{1}+q z\left(\theta_{t} \omega\right)\right)^{\mathrm{T}}, \\
R_{\mu}:\left(y_{1}, y_{2}\right)^{\mathrm{T}} & \mapsto\left(y_{1}, y_{2}-\mu y_{1}\right) .
\end{aligned}
$$

And inverse isomorphic mapping:

$$
\begin{aligned}
T_{\mu}^{-1}\left(\theta_{t} \omega\right):\left(y_{1}, y_{2}\right)^{\mathrm{T}} & \mapsto\left(y_{1}, y_{2}+\mu y_{1}-q z\left(\theta_{t} \omega\right)\right)^{\mathrm{T}}, \\
R_{\mu}^{-1}:\left(y_{1}, y_{2}\right)^{\mathrm{T}} & \mapsto\left(y_{1}, y_{2}+\mu y_{1}\right) .
\end{aligned}
$$

Then the mapping $S_{1}(t, \omega)=T_{\mu}\left(\theta_{t} \omega\right) \tilde{S}(t, \omega) T_{\mu}^{-1}\left(\theta_{t} \omega\right)$ generates a random dynamical system associated with (1.1)-(1.3); and mapping

$S(t, \omega)=R_{\mu} S_{1}(t, \omega) R_{\mu}^{-1}$ generates a random dynamical system associated with (3.2.2).

Notice that all of the above random dynamical system $S_{1}(t, \omega), S(t, \omega)$ are equivalent. Hence we only need to consider the random dynamical system 
$S(t, \omega)$.

\section{The Existence of Random Attractor}

First, we prove the random dynamical system $S(t, \omega)$ exists a bounded random absorb set, hence we let $D(E)$ be all temper subsets in $E$.

Lemma 4.1. (Lemma 3.1 of [20]) Let $V=H_{0}^{m}(\Omega) \times L^{2}(\Omega)$, for any $y=\left(y_{1}, y_{2}\right)^{\mathrm{T}} \in V$, we have

$$
(L y, y)_{E} \geq k_{1}\|y\|_{E}^{2}+k_{2}\left\|\nabla^{m} y_{2}\right\|_{0}^{2} \geq k_{1}\|y\|_{E}^{2}+k_{3}\left\|y_{2}\right\|_{0}^{2} .
$$

where $k_{1}, k_{2}$ are determined in [20], $k_{3}=\lambda_{1}^{m} k_{2}, \lambda_{1}$ is first eigenvalues of (1.1).

Lemma 4.2. Let $\psi$ is a solve of (3.2.2), then there is a bounded random compact set $\tilde{B}_{0}(\omega) \in D(E)$, such that for arbitrarily random set $B(\omega) \in D(E)$, existence a random variable $T_{B(\omega)}>0$, so that

$$
\psi\left(t, \theta_{-t} \omega\right) B\left(\theta_{-t} \omega\right) \subset B_{0}(\omega), \forall t \geq T_{B}(\omega), \omega \in \Omega .
$$

Proof. Let $\gamma$ is a solve of (3.2.3), applying the inner product of the equation (3.2.3) with $\gamma=(u, v)^{\mathrm{T}} \in E$, we discover that

$$
\frac{1}{2} \frac{\mathrm{d}}{\mathrm{d} t}\|\gamma\|_{E}^{2}+(L \gamma, \gamma)_{E}=\left(\bar{F}\left(\theta_{t} \omega, \gamma\right), \gamma\right)
$$

where

$$
\begin{aligned}
& \left(\bar{F}\left(\theta_{t} \omega, \gamma\right), \gamma\right)=\left(\nabla^{m} q(x) z\left(\theta_{t} \omega\right), \nabla^{m} u\right)+\left(-g(u)+f(x)-\left(A^{m}-\mu-1\right) q(x) z\left(\theta_{t} \omega\right), v\right) \\
& \left(\nabla^{m} q(x) z\left(\theta_{t} \omega\right), \nabla^{m} u\right) \leq \frac{1}{2}\left\|\nabla^{m} u\right\|^{2}+\frac{1}{2}\left\|\nabla^{m} q(x)\right\|^{2}\left\|z\left(\theta_{t} \omega\right)\right\|_{0}^{2}, \\
& \left(f(x)+\mu q(x) z\left(\theta_{t} \omega\right), v\right) \leq \frac{k_{3}}{2}\|v\|_{0}^{2}+\frac{1}{2 k_{3}}\left(\|f(x)\|^{2}+\mu^{2}\|q(x)\|_{0}^{2}\left\|z\left(\theta_{t} \omega\right)\right\|_{0}^{2}\right), \\
& \left(-\left(A^{m}-1\right) q(x) z\left(\theta_{t} \omega\right), v\right) \leq \frac{k_{3}}{2}\|v\|_{0}^{2}+\frac{1}{2 k_{3}}\left(\left\|A^{m} q(x)\right\|^{2}+\|q(x)\|_{0}^{2}\left\|z\left(\theta_{t} \omega\right)\right\|_{0}^{2}\right), \\
& (-g(u), v)=\left(-g(u), u_{t}+\mu u-q(x) z\left(\theta_{t} \omega\right)\right), \\
& \left(g(u), u_{t}\right)=\int_{\Omega} g(u) u_{t} \mathrm{~d} x=\frac{\mathrm{d}}{\mathrm{d} t} \int_{\Omega} G(u) \mathrm{d} x, \\
& \mu(g(u), u)=\mu \int_{\Omega} g(u) u \mathrm{~d} x \geq \mu c_{1} \int_{\Omega} G(u) \mathrm{d} x-k_{0}, k_{0} \geq 0, \\
& \left(g(u), q(x) z\left(\theta_{t} \omega\right)\right)=\int_{\Omega} g(u) q(x) z\left(\theta_{t} \omega\right) \mathrm{d} x \\
& \leq c_{0} \int_{\Omega}\left|q(x) z\left(\theta_{t} \omega\right)\right| \mathrm{d} x+c_{0} \int_{\Omega}|u|^{p}\left|q(x) z\left(\theta_{t} \omega\right)\right| \\
& \leq c_{0} \sqrt{\|\Omega\|}\|q(x)\|\left\|_{0}\right\| z\left(\theta_{t} \omega\right)\left\|_{0}+c_{0}\left(\int_{\Omega}|u|^{p+1} \mathrm{~d} x\right)^{\frac{p}{p+1}}\right\| q(x)\left\|_{L_{p+1}(\Omega)}\right\| z\left(\theta_{t} \omega\right) \|_{00} \\
& \leq C_{1}+C_{2}\|q(x)\|_{0}^{2}\left\|z\left(\theta_{t} \omega\right)\right\|_{0}^{2}+C_{3}\left(\int_{\Omega} G(u) \mathrm{d} x\right)^{\frac{p}{p+1}}\|q(x)\|_{L_{p+1}(\Omega)}\left\|z\left(\theta_{t} \omega\right)\right\|_{00} \\
& \leq C_{1}+C_{2}\|q(x)\|_{0}^{2}\left\|z\left(\theta_{t} \omega\right)\right\|_{0}^{2}+\frac{\mu C_{1}}{2} \int_{\Omega} G(u) \mathrm{d} x+C_{4}\|q(x)\|^{p+1}\left\|z\left(\theta_{t} \omega\right)\right\|_{00}^{p+1} .
\end{aligned}
$$


According to (4.1) and (4.4)-(4.10), we have

$$
\begin{aligned}
& \frac{\mathrm{d}}{\mathrm{d} t}\left(\|\gamma\|_{E}^{2}+2 \int_{\Omega} G(u) \mathrm{d} x\right)+\eta\left(\|\gamma\|_{E}^{2}+2 \int_{\Omega} G(u) \mathrm{d} x\right) \\
& \leq C_{5}+M\left\|z\left(\theta_{t} \omega\right)\right\|_{0}^{2}+N\left\|z\left(\theta_{t} \omega\right)\right\|_{0}^{p+1},
\end{aligned}
$$

where

$$
\begin{gathered}
\eta=\min \left\{2 k_{1}, \frac{\mu c_{1}}{2}\right\}, \\
M=\left(\frac{4 \mu^{2}+4}{k_{3}}+2 C_{2}\right)\|q(x)\|_{0}^{2}+\frac{4}{k_{3}}\left\|A^{m} q(x)\right\|^{2}, \\
N=2 C_{4}\|q(x)\|_{0}^{p+1} .
\end{gathered}
$$

According to Gronwall inequation, Pa.e. $\omega \in \Omega$, we have

$$
\begin{aligned}
& \|\gamma(t, \omega)\|_{E}^{2}+2 \int_{\Omega} G(u) \mathrm{d} x \\
& \leq \mathrm{e}^{-\eta t}\left(\|\gamma(0, \omega)\|_{E}^{2}+2 \int_{\Omega} G\left(u_{0}\right) \mathrm{d} x\right) \\
& \quad+\int_{0}^{t} \mathrm{e}^{-\eta(t-r)}\left(C_{5}+M\left\|z\left(\theta_{r} \omega\right)\right\|_{0}^{2}+N\left\|z\left(\theta_{r} \omega\right)\right\|_{0}^{p+1}\right) \mathrm{d} r .
\end{aligned}
$$

Because $z\left(\theta_{t} \omega\right)$ is tempered, and $z\left(\theta_{t} \omega\right)$ is continuous about $t$, according to [21], we can get a temper random variables $r_{1}: \Omega \rightarrow R^{+}$, such that $\forall t \in R, \omega \in \Omega$, we have

$$
\left\|z\left(\theta_{t} \omega\right)\right\|^{2} \leq r_{1}\left(\theta_{t} \omega\right) \leq \mathrm{e}^{\frac{\eta}{2} t} r_{1}\left(\theta_{t} \omega\right) .
$$

Substituting $\omega$ by $\theta_{-t} \omega$ in (4.12), we know

$$
\begin{aligned}
& \left\|\gamma\left(t, \theta_{-t} \omega\right)\right\|_{E}^{2}+2 \int_{\Omega} G(u) \mathrm{d} x \\
& \leq \mathrm{e}^{-\eta t}\left(\left\|\gamma\left(0, \theta_{-t} \omega\right)\right\|_{E}^{2}+2 \int_{\Omega} G\left(u_{0}\right) \mathrm{d} x\right) \\
& \quad+\int_{0}^{t} \mathrm{e}^{-\eta(t-r)}\left(C_{5}+M\left\|z\left(\theta_{r-t} \omega\right)\right\|_{0}^{2}+N\left\|z\left(\theta_{r-t} \omega\right)\right\|_{0}^{p+1}\right) \mathrm{d} r,
\end{aligned}
$$

where

$$
\begin{aligned}
& \int_{0}^{t} \mathrm{e}^{-\eta(t-r)}\left(C_{5}+M\left\|z\left(\theta_{r-t} \omega\right)\right\|_{0}^{2}+N\left\|z\left(\theta_{r-t} \omega\right)\right\|_{0}^{p+1}\right) \mathrm{d} r \\
& =\int_{-t}^{0} \mathrm{e}^{\eta t}\left(C_{5}+M\left\|z\left(\theta_{\tau} \omega\right)\right\|_{0}^{2}+N\left\|z\left(\theta_{\tau} \omega\right)\right\|_{0}^{p+1}\right) \mathrm{d} \tau \\
& \leq \frac{C_{5}}{\eta}+\frac{2}{\eta} M r_{1}(\omega)+\frac{2}{\eta} N r_{1}^{\frac{p+1}{2}}(\omega) .
\end{aligned}
$$

Because $\psi_{0}\left(\theta_{-t} \omega\right) \in B\left(\theta_{-t} \omega\right)$ is tempered, and $\left\|z\left(\theta_{-t} \omega\right)\right\|$ is also tempered, hence we let

$$
R_{0}^{2}(\omega)=\frac{C_{5}}{\eta}+\frac{2}{\eta} M r_{1}(\omega)+\frac{2}{\eta} N r_{1}^{\frac{p+1}{2}}(\omega),
$$

then $R_{0}^{2}(\omega)$ is also tempered, $\hat{B}_{0}=\left\{\gamma \in E:\|\gamma\|_{E} \leq R_{2}(\omega)\right\}$ is called a random 
absorb set, and because of

$\tilde{S}\left(t, \theta_{-t} \omega\right) \gamma_{0}\left(\theta_{-t} \omega\right)=\psi\left(t, \theta_{-t} \omega\right)\left(\gamma_{0}\left(\theta_{-t} \omega\right)+\left(0, q(x) z\left(\theta_{-t} \omega\right)\right)^{\mathrm{T}}\right)-\left(0, q(x) z\left(\theta_{t} \omega\right)\right)^{\mathrm{T}}$,

so let

$\tilde{B}_{0}(\omega)=\left\{\psi \in E:\|\psi\|_{E} \leq R_{0}(\omega)+\left\|q(x) z\left(\theta_{t} \omega\right)\right\|=\overline{R_{0}}(\omega)\right\}$, then $\tilde{B}_{0}(\omega)$ is a random absorb set of $\psi(t, \omega)$, and $\tilde{B}_{0}(\omega) \in D(E)$.

Next, we will prove the random dynamical system $S(t, \omega)$ has a compact absorb set

Lemma 4.3. For $\forall B(\omega) \in D(E)$, let $\psi(t)$ be a solve of (3.2.2), initial value $\psi_{0}=\left(u_{0}, u_{1}+\mu u_{0}\right)^{\mathrm{T}} \in B$, we decompose $\psi=\psi^{1}+\psi^{2}$, where $\psi^{1}, \psi^{2}$ satisfy

$$
\begin{gathered}
\left\{\begin{array}{l}
\mathrm{d} \psi^{1}+L \psi^{1} \mathrm{~d} t=0, \\
\psi_{0}^{1}(\omega)=\left(u_{0}, u_{1}+\mu u_{0}\right)^{\mathrm{T}},
\end{array}\right. \\
\left\{\begin{array}{l}
\mathrm{d} \psi^{2}+L \psi^{2} \mathrm{~d} t=0, \\
\psi_{0}^{2}(\omega)=0 .
\end{array}\right.
\end{gathered}
$$

Then

$$
\left\|\psi^{1}\left(t, \theta_{-t} \omega\right)\right\|_{E}^{2} \rightarrow 0(t \rightarrow \infty), \forall \psi_{0}\left(\theta_{-t} \omega\right) \in B\left(\theta_{-t} \omega\right),
$$

and exist a temper random radius $R_{1}(\omega)$, such that $\forall \omega \in \Omega$, satisfy

$$
\left\|A^{\frac{m}{4}} \psi^{2}\left(t, \theta_{-t} \omega\right)\right\|_{E} \leq R_{1}(\omega) .
$$

Proof. Let $\gamma=\gamma^{1}+\gamma^{2}=\left(u^{1}, u_{t}^{1}+\mu u^{1}\right)^{\mathrm{T}}+\left(u^{2}, u_{t}^{2}+\mu u^{2}-q z\left(\theta_{t} \omega\right)\right)^{\mathrm{T}}$ be a solve of (3.2.3), according to (4.17) and (4.18), we know $\gamma^{1}, \gamma^{2}$ meet separately

$$
\begin{gathered}
\left\{\begin{array}{l}
\mathrm{d} \gamma_{t}^{1}+L \gamma^{1} \mathrm{~d} t=0, \\
\gamma_{0}^{1}(\omega)=\left(u_{0}, u_{1}+\mu u_{0}-q z\left(\theta_{t} \omega\right)\right)^{\mathrm{T}},
\end{array}\right. \\
\left\{\begin{array}{l}
\mathrm{d} \gamma_{t}^{2}+L \gamma^{2} \mathrm{~d} t=\bar{F}\left(\theta_{t} \omega, \gamma\right), \\
\gamma_{0}^{2}(\omega)=0 .
\end{array}\right.
\end{gathered}
$$

Taking inner product (4.21) with $\gamma^{1}=\left(u^{1}, u_{t}^{1}+\mu u^{1}\right)^{\mathrm{T}}$, we have

$$
\frac{1}{2} \frac{\mathrm{d}}{\mathrm{d} t}\left\|\gamma^{1}\right\|_{E}^{2}+\left(L \gamma^{1}, \gamma^{1}\right)=0,
$$

according to Lemma 4.1 and Gronwall inequality, we have

$$
\left\|\gamma^{1}(t, \omega)\right\|_{E}^{2} \leq \mathrm{e}^{-2 \xi t}\left\|\gamma^{1}(0, \omega)\right\|_{E}^{2},
$$

substituting $\omega$ by $\theta_{-t} \omega$, and $z\left(\theta_{-t} \omega\right) \in B$ is tempered, then

$$
\left\|\gamma^{1}\left(t, \theta_{-t} \omega\right)\right\|_{E}^{2} \leq \mathrm{e}^{-2 \xi t}\left\|\gamma^{1}\left(0, \theta_{-t} \omega\right)\right\|_{E}^{2} \rightarrow 0(t \rightarrow \infty), \forall \gamma\left(0, \theta_{-t} \omega\right) \in B .
$$

So, (4.19) is hold. Taking inner product (4.22) with

$$
\begin{aligned}
A \frac{m}{2} \gamma^{2}=A & \frac{m}{2}\left(u^{2}, u_{t}^{2}+\mu u^{2}-q(x) z\left(\theta_{t} \omega\right)\right)^{\mathrm{T}} \text {, we have } \\
& \frac{1}{2} \frac{\mathrm{d}}{\mathrm{d} t}\left\|A^{\frac{m}{4}} \gamma^{2}\right\|_{E}^{2}+\left(L \gamma^{2}, A \frac{m}{2} \gamma^{2}\right)=\left(\bar{F}\left(\gamma, \theta_{t} \omega\right), A \frac{m}{2} \gamma^{2}\right),
\end{aligned}
$$


according to Lemma 4.1, Lemma 4.2, (4.24) and Young inequality, we have

$$
\begin{aligned}
& \frac{\mathrm{d}}{\mathrm{d} t}\left(\left\|A^{\frac{m}{4}} \gamma^{2}\right\|_{E}^{2}+2 \int_{\Omega} A^{\frac{m}{2}} G\left(u^{2}\right) \mathrm{d} x\right)+\eta\left(\left\|A^{\frac{m}{4}} \gamma^{2}\right\|_{E}^{2}+2 \int_{\Omega} A^{\frac{m}{2}} G\left(u^{2}\right) \mathrm{d} x\right) \\
& \leq C_{5} R_{0}^{2}\left(\theta_{t} \omega\right)+M_{1}\left\|z\left(\theta_{t} \omega\right)\right\|_{0}^{2}+N_{2}\left\|z\left(\theta_{t} \omega\right)\right\|_{0}^{p+1}, \forall t \geq T_{B(\omega)},
\end{aligned}
$$

where $T_{B(\omega)}, R_{0}(\omega)$ are given by Lemma 4.2 , and

$$
M_{1}=\left(\frac{4 \mu^{2}+4}{k_{3}}+2 C_{2}\right)\left\|A^{\frac{m}{4}} q(x)\right\|^{2}+\frac{4}{k_{3}}\left\|A^{m} q(x)\right\|^{2},
$$

$N_{1}=C_{4}\left\|A^{\frac{m}{4}} q(x)\right\|^{p+1}$. Due to Gronwall inequality, and substituting $\omega$ by $\theta_{-t} \omega$, we have

$$
\begin{aligned}
& \left\|A^{\frac{m}{4}} \gamma^{2}\left(t, \theta_{-t} \omega\right)\right\|_{E}^{2}+2 \int_{\Omega} A^{\frac{m}{2}} G\left(u^{2}\right) \mathrm{d} x \\
& \leq \int_{0}^{t} \mathrm{e}^{-\eta(t-r)}\left(C_{5} R_{0}^{2}\left(\theta_{r-t} \omega\right)+M_{1}\left\|z\left(\theta_{r-t} \omega\right)\right\|_{0}^{2}+N_{2}\left\|z\left(\theta_{r-t} \omega\right)\right\|_{0}^{p+1}\right) \mathrm{d} r, \forall t \geq T_{B(\omega)} .
\end{aligned}
$$

According to (4.14) and (4.16), then

$$
\begin{aligned}
& \int_{0}^{t} \mathrm{e}^{-\eta(t-r)}\left(C_{5} R_{0}^{2}\left(\theta_{r-t} \omega\right)+M_{1}\left\|z\left(\theta_{r-t} \omega\right)\right\|_{0}^{2}+N_{2}\left\|z\left(\theta_{r-t} \omega\right)\right\|_{0}^{p+1}\right) \mathrm{d} r \\
& =\int_{-t}^{0}\left(C_{5} R_{0}^{2}\left(\theta_{\tau} \omega\right)+M_{1}\left\|z\left(\theta_{\tau} \omega\right)\right\|_{0}^{2}+N_{2}\left\|z\left(\theta_{\tau} \omega\right)\right\|_{0}^{p+1}\right) \mathrm{d} r \\
& =\frac{C_{5}^{2}}{\eta^{2}}+\left(\frac{2}{\eta} M_{1}+\frac{4 C_{5}}{\eta^{2}} M\right) r_{1}(\omega)+\left(\frac{2}{\eta} N_{1}+\frac{8}{(p+1) \eta} N\right) r_{1}^{\frac{p+1}{2}}(\omega) .
\end{aligned}
$$

Let

$$
\bar{R}_{1}^{2}(\omega)=\frac{C_{5}^{2}}{\eta^{2}}+\left(\frac{2}{\eta} M_{1}+\frac{4 C_{5}}{\eta^{2}} M\right) r_{1}(\omega)+\left(\frac{2}{\eta} N_{1}+\frac{8}{(p+1) \eta} N\right) r_{1}^{\frac{p+1}{2}}(\omega) .
$$

Then $\bar{R}_{1}^{2}(\omega)$ is tempered, and because

$\tilde{S}\left(t, \theta_{-t} \omega\right) \gamma_{0}\left(\theta_{-t} \omega\right)=\psi\left(t, \theta_{-t} \omega\right)\left(\gamma_{0}\left(\theta_{-t} \omega\right)+\left(0, q(x) z\left(\theta_{-t} \omega\right)\right)^{\mathrm{T}}\right)-\left(0, q(x) z\left(\theta_{t} \omega\right)\right)^{\mathrm{T}}$,

hence, we set

$$
\begin{aligned}
& R_{1}(\omega)=\bar{R}_{1}(\omega)+\left\|A^{\frac{m}{4}} q(x) z\left(\theta_{t} \omega\right)\right\| \text {, then, for } \forall \omega \in \Omega \text {, we have } \\
& \left\|A^{\frac{m}{4}} \psi^{2}\left(t, \theta_{-t} \omega\right)\right\|_{E} \leq R_{1}(\omega) \text {, and } R_{1}(\omega) \text { is tempered. }
\end{aligned}
$$

Lemma 4.4. (3.2.2) the identified stochastic dynamical system $S(t, \omega), t \geq 0$, while $t=0$, Pa.e. $\omega \in \Omega$ exist a compact attracting set $K(\omega) \subset E$.

Proof. Let $K(\omega)$ be a closed ball, radius $R_{1}(\omega)$ in space $D\left(A^{\frac{3}{4} m}\right) \times D\left(A^{\frac{m}{4}}\right)$, because $D\left(A^{\frac{3}{4} m}\right) \times D\left(A^{\frac{m}{4}}\right) \mapsto E$, so $K(\omega)$ is a compact set in $E$, for arbitrarily temper random set $B(\omega)$, for $\forall \psi\left(t, \theta_{-t} \omega\right) \in B$, according to Lemma 4.3, $\psi^{2}=\psi-\psi^{1} \in K(\omega)$, so for $\forall t \geq T_{B(\omega)}>0$, we have 


$$
\begin{aligned}
& d_{E}\left(S\left(t, \theta_{-t} \omega\right) B\left(\theta_{-t} \omega\right), K(\omega)\right) \\
& =\inf _{\beta(t) \in K(\omega)}\left\|\psi\left(t, \theta_{-t} \omega\right)-\beta(t)\right\|_{E}^{2} \leq\left\|\psi^{1}\left(t, \theta_{-t} \omega\right)\right\|_{E}^{2} \\
& \leq \mathrm{e}^{-2 \eta t}\left\|\psi_{0}^{1}\left(t, \theta_{-t} \omega\right)\right\|_{E}^{2} \rightarrow 0,(t \rightarrow \infty) .
\end{aligned}
$$

Theorem 4.1. The random dynamical system $S(t, \omega), t \geq 0$ has a unique random attractor $A(\omega)$ in $E$, where

$$
A(\omega)=\bigcap_{t>0} \bar{\tau} S\left(t, \theta_{-\tau} \omega, K\left(\theta_{-\tau} \omega\right)\right), \omega \in \Omega,
$$

in which $K(\omega)$ is a tempered random compact attracting for $S(t, \omega)$.

\section{References}

[1] Yang, Z.-J. (2007) Long-Time Behavior of the Kirchhoff Type Equation with Strong Damping in $R^{N}$. Journal of Differential Equations, 242, 269-286.

[2] Yang, Z.J. and Ding, P.Y. (2016) Longtime Dynamics of the Kirchhoff Equation with Strong Damping and Critical Nonlinearity on $R^{N}$. Journal of Mathematical Analysis and Applications, 434, 1826-1851.

[3] Chueshov, I. (2012) Longtime Dynamics of Kirchhoff Wave Models with Strong Nonlinear Damping. Journal of Differential Equations, 252, 1229-1262.

[4] Ono, K. (1997) On Global Existence, Asymptotic Stability and Blowing up of Solutions for Some Degenerate Non-Linear Wave Equations of Kirchhoff Type with a Strong Dissipation. Mathematical Methods in the Applied Sciences, 20, 151-177. https://doi.org/10.1002/(SICI)1099-1476(19970125)20:2<151::AID-MMA851>3.0.C $\mathrm{O} ; 2-0$

[5] Wang, Z.J., Zhou, S.F. and Gu, A.H. (2011) Random Attractor for a Stochastic Damped Wave Equation with Multiplicative Noise on Unbounded Domains. Nonlinear Analysis: Real World Applications, 12, 3468-3482.

[6] Xu, G.G., Wang, L.B. and Lin, G.G. (2013) Global Random Attractor for the Strongly Damped Stochastic Wave Equation with White Noise and Nonlinear Term. Theoretical Mathematics \& Applications, 3, 111-122.

[7] Zhong, C.K., Yang, M.H. and Sun, C.Y. (2006) The Existence of Global Attractors for the Norm-to-Weak Continuous Semigroup. Journal of Differential Equations, 223, 367-399.

[8] Wang, Z.J., Zhou, S.F. and Gu, A.H. (2012) Random Attractor of the Stochastic Strongly Damped Wave Equation. Communications in Nonlinear Science and $\mathrm{Nu}$ merical Simulation, 17, 1649-1658.

[9] Arnold, L. (1998) Random Dynamical Systems. Springer-Verlag, Berlin. https://doi.org/10.1007/978-3-662-12878-7

[10] Chueshov, I. (2002) Monotone Random Systems Theory and Applications. Springer-Verlag, Berlin. https://doi.org/10.1007/b83277

[11] Guo, B. and Pu, X. (2009) Random Infinite Dimensional Dynamical System. Beijing University of Aeronautics and Astronautics Press, Beijing.

[12] Lu, D. (1986) Stochastic Process and Its Application. Tsinghua University Press, Beijing.

[13] Fan, X. (2010) Attractors for a Damped Stochastic Wave Equation of Sine-Gordon Type with Sub-Linear Multiplicative Noise. Stochastic Analysis and Applications, 
24, 767-793. https://doi.org/10.1080/07362990600751860

[14] Pazy, A. (1983) Semi-Groups of Linear Operators and Applications to Partial Differential Equations. Springer Verlag, New York.

[15] Massatt, P. (1983) Limiting Behaviour for a Strong Damped Nonlinear Wave Equation. Journal of Differential Equations, 48, 334-349.

[16] Crauelh, N.D. (1988) Random Nonlinear Wave Equations: Smoothness of the Solutions. Probability Theory and Related Fields, 79, 469-508.

https://doi.org/10.1007/BF00318783

[17] Prato, G.D. and Zabczyk, J. (1992) Stochastic Equations in Infinite Dimensions. Cambridge University Press, London, 115-236.

[18] Lin, G. (2011) Nonlinear Evolution Equation. Yunnan University Press, Kunming.

[19] Qin, C. and Lin, G. (2010) Random Attractor of the Weakly Damped Stochastic Kirchhoff Equations. Journal of Yunnan University(Nature), 32, 101-108.

[20] Chen, L., Wang, W. and Lin, G. (2016) Exponential Attractor and Inertial Manifolds for the Higher-Order Nonlinear Kirchhoff-Type Equation. International Journal of Modern Communication Technologies and Research, 4, 6-12.

[21] Zhao, C. and Zhou, S. (2009) Sufficient Conditions for the Existence of Global Random Attractors for Stochatic Lattice Dunamical Systems and Applications. Journal of Mathematical Analysis and Applications, 354, 78-95.

Submit or recommend next manuscript to SCIRP and we will provide best service for you:

Accepting pre-submission inquiries through Email, Facebook, LinkedIn, Twitter, etc. A wide selection of journals (inclusive of 9 subjects, more than 200 journals)

Providing 24-hour high-quality service

User-friendly online submission system

Fair and swift peer-review system

Efficient typesetting and proofreading procedure

Display of the result of downloads and visits, as well as the number of cited articles

Maximum dissemination of your research work

Submit your manuscript at: http://papersubmission.scirp.org/

Or contactijmnta@scirp.org 